\title{
Incidence of Interval Colorectal Cancer Among Inflammatory Bowel Disease Patients Undergoing Regular Colonoscopic Surveillance
}

\author{
Erik Mooiweer, ${ }^{\star}$ Andrea E. van der Meulen-de Jong, ${ }^{\ddagger}$ Cyriel Y. Ponsioen, ${ }^{\S}$ \\ C. Janneke van der Woude," Ad A. van Bodegraven, " Jeroen M. Jansen, ${ }^{*}$ Nofel Mahmmod, ${ }^{* *}$ \\ Willemijn Kremer, ${ }^{*}$ Peter D. Siersema, ${ }^{*}$ and Bas Oldenburg, ${ }^{*}$ on behalf of the Dutch Initiative \\ on Crohn's and Colitis
}

\begin{abstract}
*Department of Gastroenterology and Hepatology, University Medical Center Utrecht, Utrecht, The Netherlands; ${ }^{\ddagger}$ Department of Gastroenterology and Hepatology, Leiden University Medical Center, Leiden, The Netherlands;

${ }^{\S}$ Department of Gastroenterology and Hepatology, Amsterdam Medical Center, Amsterdam, The Netherlands; "Department of Gastroenterology and Hepatology, Erasmus Medical Center Rotterdam, Rotterdam, The Netherlands; "Department of Gastroenterology and Hepatology, Vrije Universiteit Medical Center Amsterdam, Amsterdam, The Netherlands; "Department of Gastroenterology and Hepatology, Onze Lieve Vrouwe Gasthuis Amsterdam, Amsterdam, The Netherlands; ${ }^{* *}$ Department of Gastroenterology and Hepatology, St Antonius Hospital Nieuwegein, Nieuwegein, The Netherlands
\end{abstract}

BACKGROUND \& AIMS:

Surveillance is recommended for patients with long-term inflammatory bowel disease because they have an increased risk of colorectal cancer (CRC). To study the effectiveness of surveillance, we determined the incidence of CRC after negative findings from surveillance colonoscopies (interval CRC).

METHODS:
We collected data from 1273 patients with ulcerative colitis or Crohn's disease, enrolled in a sur- veillance program at 7 hospitals in The Netherlands, who underwent 4327 surveillance colonoscopies from January 1, 2000, through January 1, 2014. Patients were followed up from their first surveillance colonoscopy until the last surveillance colonoscopy, colectomy, or CRC. Factors that might have contributed to the occurrence of CRC were categorized as inadequate procedures (ie, inadequate bowel preparation), inadequate surveillance (CRC occurring outside the appropriate surveillance interval), or inadequate management of dysplasia (CRC diagnosed in the same colonic segment as a previous diagnosis of dysplasia). The remaining CRC cases were classified as true interval CRCs.

RESULTS:

CRC was diagnosed in 17 patients (1.3\%), with an incidence of 2.5 per 1000 years of follow-up evaluation. Factors that might account for the occurrence of CRC were identified in 12 patients $(70 \%)$. These were inadequate colonoscopies in 4 patients $(24 \%)$, inadequate surveillance intervals in 9 patients (53\%), and inadequate management of dysplasia in 2 patients (12\%). The remaining 5 cases of CRC (30\%) were classified as true interval CRCs.

CONCLUSIONS:

In a retrospective analysis of patients with inflammatory bowel disease participating in a surveillance program, the incidence of CRC was only $1 \%$, which supports the implementation of longer surveillance intervals. However, the fact that $30 \%$ of CRC cases were interval cancers indicates the need for variable surveillance intervals based on risk factors for CRC.

Keywords: Inflammatory Bowel Disease; Surveillance; Interval Colorectal Cancer.

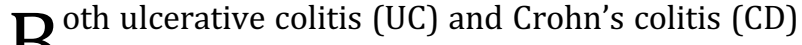

$\mathbf{B}$ are associated with an increased risk of developing colorectal cancer (CRC). ${ }^{1,2}$ Therefore, regular endoscopic surveillance is recommended, which aims to achieve a reduction in CRC-related deaths by detecting and treating premalignant lesions (dysplasia) and early stage asymptomatic CRC. ${ }^{3,4}$ There has been much debate regarding whether this strategy is (cost) effective, mainly
Abbreviations used in this paper: AGA, American Gastroenterological Association; BSG, British Society of Gastroenterology; CD, Crohn's disease; CRC, colorectal cancer; DTC, diagnosis treatment combinations; HGD, high-grade dysplasia; IBD, inflammatory bowel disease; LGD, low-grade dysplasia; PSC, primary sclerosing cholangitis; UC, ulcerative colitis.

(Q) Most current article

(C) 2015 by the AGA Institute $1542-3565 / \$ 36.00$

http://dx.doi.org/10.1016/j.cgh.2015.04.183 
because the evidence supporting the effectiveness of surveillance is limited. ${ }^{5,6}$

The lack of solid evidence for CRC surveillance in IBD patients also is reflected by the differences in the recommended surveillance intervals of the recently updated British Society of Gastroenterology (BSG) and American Gastroenterological Association (AGA) surveillance guidelines. ${ }^{3,4}$ The BSG guideline adopted a risk-stratified approach based on known risk factors for IBD-associated CRC with corresponding surveillance intervals of 1,3 , and 5 years, whereas the authors of the AGA guidelines stated that optimal surveillance intervals cannot be defined clearly and therefore recommend that surveillance should be performed every 1 to 3 years. Determining the optimal surveillance interval is important because a shorter interval leads to unnecessary high costs and burdens the patient, whereas a longer interval could decrease the effectiveness of surveillance and may result in an increased incidence of CRC detected between 2 surveillance colonoscopies (interval CRC).

Because a direct prospective comparison between different surveillance intervals probably never will be performed, data on the occurrence of interval CRCs among IBD patients undergoing regular surveillance could provide vital information on whether current surveillance protocols are effective. ${ }^{7}$ The occurrence of interval CRCs among IBD patients undergoing surveillance largely is unknown, although 1 study reported that as many as $50 \%$ of the CRCs diagnosed during the surveillance program were interval CRCs. ${ }^{8}$ These data date back to the 1970s, however, and it is not clear if these results can be extrapolated to the present clinical situation.

The aim of the current study therefore was to establish the incidence of interval CRCs in a cohort of IBD patients undergoing regular colonoscopic surveillance. Furthermore, we aimed to identify procedure-related factors potentially explaining the occurrence of CRC.

\section{Methods}

\section{Patients}

All patients with a diagnosis of CD or UC from 5 university hospitals and 2 general hospitals were identified using the diagnosis treatment combinations (DTCs) for IBD. DTCs are based on the International Classification of Diseases codes and can be considered the Dutch version of the Diagnosis Related Groups, as used in other countries (eg, the United States).

The medical records and endoscopy reports of all patients with a DTC code for CD or UC were reviewed to confirm the IBD diagnosis and to assess whether patients had a valid indication for surveillance colonoscopy according to the new AGA and BSG guidelines. Because the new BSG and AGA guidelines do not concur with regard to the beginning of surveillance (10 and 8 years after the onset of colitis symptoms, respectively), we considered patients with a disease duration of at least 8 years to be eligible for surveillance. Patients with colitis and a concomitant diagnosis of primary sclerosing cholangitis (PSC) were considered to have an indication for surveillance immediately after diagnosis whereas patients with proctitis or proctosigmoiditis (UC patients) or with involvement of less than $30 \%$ of the colonic mucosa (CD patients) were considered to have no indication for surveillance and were excluded.

Furthermore, patients with any previous diagnosis of neoplasia (other than discrete solitary sessile or pedunculated polyps suggestive of sporadic adenomas and containing adenomatous tissue on histology) or (sub) total colectomy before the first surveillance colonoscopy were excluded.

Demographic and clinical data were collected from the medical records and included date of IBD diagnosis, type of IBD, disease extent before the start of surveillance, family history of CRC, medication use, and a concomitant diagnosis of PSC. In UC and IBD-unclassified patients, disease extent was defined as either left-sided or extensive (inflammation distal or proximal to the splenic flexure, respectively). In patients with $\mathrm{CD}$, involvement of 3 or more anatomic parts of the colon was considered extensive disease, whereas involvement of 1 or 2 sections was considered limited disease.

Patients were followed up from the date of the first surveillance colonoscopy after January 1, 2000, until the last surveillance colonoscopy before January 7, 2014, (sub)total colectomy, or the diagnosis of CRC.

\section{Surveillance Colonoscopies}

During the study period all participating centers performed surveillance in accordance with international guidelines (ie, complete colonic inspection with or without the use of chromoendoscopy and biopsy sampling of all areas suspicious for neoplasia, as well as sampling of 4 random biopsy specimens every $10 \mathrm{~cm}$ when chromoendoscopy was not performed).

All endoscopy reports were reviewed to confirm that patients underwent at least 2 surveillance colonoscopies. A surveillance colonoscopy was defined as a procedure with the clear intention to detect neoplasia (explicitly stated as the indication for the colonoscopy and/or by the use of chromoendoscopy or taking 4-quadrant random biopsy specimens every $10 \mathrm{~cm}$ ).

Potential quality indicators such as cecal intubation and the number of random biopsy specimens were collected from the endoscopy reports. Insufficient bowel preparation was recorded if this was stated as such in the endoscopy report. Furthermore, known endoscopic risk factors for IBD-associated CRC were collected such as the extent and severity of inflammation and the presence of post-inflammatory polyps or strictures.

For all lesions that were detected during the follow-up period and suspected of containing dysplasia, the location, endoscopic description, and treatment were recorded. 
Based on the pathology report, lesions were categorized as nondysplastic, indefinite for dysplasia, low-grade dysplasia (LGD), high-grade dysplasia (HGD), or colorectal cancer (CRC). Endoscopically visible lesions containing LGD were subdivided into adenoma-like masses (discrete solitary sessile or pedunculated polyps resembling sporadic adenomas and containing adenomatous tissue on histology) and non-adenoma-like masses (all other endoscopic descriptions; ie, plaque-like lesions, irregular masses).

\section{Colorectal Cancer Characteristics}

Each diagnosis of CRC within the surveillance program was categorized according to its most probable explanation, adopted from similar studies on sporadic CRC. ${ }^{9}$ Procedure-related CRCs were defined as inadequate bowel preparation, incomplete intubation, or fewer than 10 random biopsy specimens sampled during the surveillance colonoscopy before the CRC diagnosis. Inadequate surveillance was defined as CRC occurring outside the appropriate surveillance interval. Because the 2002 BSG guidelines were followed during the study period, we considered a 1-year surveillance interval appropriate for patients with prior dysplasia or a concomitant diagnosis of PSC and a 2-year surveillance interval for the remaining patients. We also stratified patients in the 1-year, 3-year, and 5-year surveillance intervals based on the risk factors described in the new BSG guidelines. Inadequate dysplasia management was defined as CRC diagnosed in the same colonic segment as a previous diagnosis of dysplasia.

\section{Definition of Interval Colorectal Cancer}

All cases of CRC detected within the appropriate surveillance interval, after an adequately performed surveillance colonoscopy, were considered interval cancers. In other words, these were cases of CRC that could be explained only by either missed lesions during the preceding colonoscopy or rapid progression to cancer within the surveillance interval. In a second analysis we defined interval CRCs as advanced stage CRC (Dukes stages C or D) diagnosed within the appropriate surveillance interval, in accordance with the definition used by Rutter et al. ${ }^{8}$

\section{Results}

\section{Surveillance Cohort}

A total of 1273 IBD patients were identified who were enrolled in a surveillance program, of whom 434 patients had CD (34\%), 804 had UC (63\%), and 35 had IBD unclassified (3\%). Baseline characteristics are shown in Table 1.

During a mean follow-up period of 5.3 years $( \pm 3.0 \mathrm{y})$, 4327 surveillance colonoscopies were performed in the study population, comprising 6823 years of follow-up evaluation. Surveillance was performed with random
Table 1. Baseline Characteristics of Patients Undergoing Surveillance

\begin{tabular}{lc}
\hline & $\mathrm{N}(\%)$ \\
\hline Patients & 1273 \\
Male sex & $676(53)$ \\
IBD diagnosis & \\
Ulcerative colitis & $804(63)$ \\
$\quad$ Distal splenic flexure & $269(33)$ \\
$\quad$ Proximal splenic flexure & $456(57)$ \\
$\quad$ Unknown & $79(10)$ \\
Crohn's colitis & $434(34)$ \\
$\quad$ Segmental colitis, $<50 \%$ & $159(37)$ \\
$\quad$ Segmental colitis, $>50 \%$ & $228(52)$ \\
$\quad$ Unknown & $47(11)$ \\
Indeterminate colitis & $35(3)$ \\
$\quad$ Segmental colitis, $<50 \%$ & $16(46)$ \\
$\quad$ Segmental colitis, $>50 \%$ & $18(51)$ \\
$\quad$ Unknown & $1(3)$ \\
Age at first surveillance colonoscopy, mean $( \pm S D), y$ & $45.8( \pm 12.9)$ \\
Duration of IBD at first surveillance colonoscopy, & $17.4( \pm 3.9)$ \\
$\quad$ mean ( \pm SD), $y$ & \\
Concomitant diagnosis of PSC & $173(14)$ \\
Duration of follow-up evaluation, mean $( \pm$ SD), $y$ & $5.3( \pm 3.0)$
\end{tabular}

biopsy sampling in 3887 procedures (90\%), during which a mean of 27 biopsy specimens $( \pm 12)$ were sampled (Table 2). The remaining 440 surveillance procedures $(10 \%)$ were performed using chromoendoscopy with targeted biopsies. The cecal intubation rate was $99 \%$, and bowel preparation was deemed adequate in $87 \%$ of procedures.

\section{Incidence of Dysplasia During Follow-Up Evaluation}

LGD was detected during 354 surveillance colonoscopies in 265 patients (21\%) during the follow-up

Table 2. Endoscopic Characteristics

\begin{tabular}{lc}
\hline & $\mathrm{N}(\%)$ \\
\hline Total number of surveillance colonoscopies & $4327(100)$ \\
WLE + random biopsies & $3887(90)$ \\
Chromoendoscopy & $440(10)$ \\
Surveillance colonoscopies, median (range) & $2(1-11)$ \\
Cecal intubation & $4288(99)$ \\
Suboptimal bowel preparation & $566(13)$ \\
Biopsy specimens per colonoscopy, mean $( \pm \mathrm{SD})$ & $27( \pm 12)$ \\
Endoscopic inflammation & \\
No active inflammation & 2847 \\
Mild & 1182 \\
Moderate to severe & 298 \\
Histologic inflammation & \\
No active inflammation & 2383 \\
Mild & 1429 \\
Moderate or severe & 276 \\
Unknown & 239 \\
Postinflammatory polyps & 926
\end{tabular}

WLE, white-light endoscopy 
evaluation, comprising 482 lesions with LGD. These lesions were classified as 339 adenomas, 43 non-adenomalike lesions, and 100 episodes of flat dysplasia, originating from a total of 107,969 random biopsy specimens. The incidence of LGD was 52 per 1000 years of follow-up evaluation.

HGD was detected during 19 surveillance colonoscopies in 19 patients (1.5\%), with an incidence of 2.8 per 1000 years of follow-up evaluation. HGD was found in a random biopsy specimen in 2 patients $(0.2 \%)$, and in a lesion in the remaining 17 patients $(1.3 \%)$.

\section{Incidence of Colorectal Cancer During Follow-Up Evaluation}

In total, 18 CRCs were diagnosed in 17 patients (1.3\%) at a median age of 55 years with an incidence rate of 2.5 per 1000 years of follow-up evaluation (Table 3). CRC was detected during a scheduled surveillance colonoscopy in 14 patients. The remaining 3 CRCs were detected during colonoscopy for an indication other than surveillance, as an incidental finding during ileocecal resection, or after analysis of liver metastases with an unknown primary tumor.

A schematic presentation of all surveillance colonoscopies and diagnoses of dysplasia before the CRC diagnosis for each individual patient are shown in Figure 1. The median interval between the last surveillance colonoscopy and the CRC diagnosis was 22 months (range, 9-42 mo).

\section{Factors Potentially Explaining the Occurrence of Colorectal Cancer}

An inadequate colonoscopy was identified as the most likely cause in 4 patients (24\%) (Figure 2). An inadequate surveillance interval was identified as an etiologic factor in 9 patients (53\%) according to the previous BSG and AGA guidelines, and in 6 patients (35\%) when patients were stratified according to the surveillance intervals of the updated BSG guideline.

Table 3. Neoplasia During Follow-Up Evaluation, Based on the Maximal Grade of Dysplasia

\begin{tabular}{lc}
\hline & $\mathrm{N}(\%)$ \\
\hline Surveillance colonoscopies & $4327(100)$ \\
Colonoscopies with neoplasia & $438(10)$ \\
Colonoscopies with IFD & $69(2)$ \\
Lesions containing LGD & $482(11)$ \\
Adenoma-like mass & $339(70)$ \\
Flat dysplasia & $100(21)$ \\
Non-adenoma-like mass & $43(9)$ \\
HGD & $19(0.4)$ \\
CRC & $17(0.4)$ \\
& \\
\hline
\end{tabular}

IFD, indefinite for dysplasia.
Two patients $(12 \%)$ had either flat LGD originating from random biopsy specimens $(n=1)$ or LGD in an endoscopically resected lesion $(n=1)$ during the surveillance procedure directly before the CRC diagnosis, which was located in the same colonic segment as the CRC (inadequate dysplasia management).

Five cases of CRC (30\%) could be classified as interval CRC according to our definition of CRC diagnosed within the appropriate surveillance interval after an adequately performed surveillance colonoscopy, resulting in an incidence of 0.2 interval CRCs per 1000 years of follow-up evaluation. Interestingly, all 5 cases were advanced stage CRC, Dukes C or D, and therefore could be classified as interval cancers according to our second definition for interval CRC as well. Because the remaining 3 cases of CRC diagnosed at an advanced stage all were diagnosed outside the appropriate surveillance interval, the rate of interval CRC was equal for both definitions. Three cases of interval CRC were identified during a regular surveillance colonoscopy at the appropriate interval, 1 during a colonoscopy for abdominal pain and 1 for liver metastases with an unknown primary tumor. Three interval CRCs (60\%) were located in the right colon, as compared with 8 of the remaining 12 CRCs (67\%). Three patients with interval CRC had UC (60\%), none had a concomitant diagnosis of PSC, and 2 patients had postinflammatory polyps (40\%).

\section{Discussion}

This study shows that the incidence of CRC among IBD patients enrolled in a surveillance program is low compared with previous studies, with only 17 cancers detected during 6823 years of follow-up evaluation. ${ }^{8}$

The majority of CRC cases (70\%) could be explained by an inadequate surveillance procedure before the CRC diagnosis (ie, inadequate bowel preparation), inadequate surveillance interval, or inadequate dysplasia management. This suggests that the incidence of CRC could have been even lower if surveillance was performed strictly according to the guidelines in all patients. Several studies have highlighted the problem of adherence to the CRC surveillance guidelines in patients with long-standing IBD. ${ }^{10-13}$ The relatively large number of potentially preventable CRCs in the present surveillance cohort stresses the importance of increasing the awareness of both patients and gastroenterologists for adequately performed surveillance at the appropriate interval.

The CRC incidence of 2.5 per 1000 person-years of follow-up evaluation in the current study is substantially lower than the incidence of 5.9 per 1000 person-years of follow-up evaluation reported by Rutter et $\mathrm{al}^{8}{ }^{8}$ using a large surveillance cohort, despite a similar study design. This probably can be explained by the fact that our study included data from the year 2000 onward, whereas the Rutter et $\mathrm{al}^{8}$ cohort dates back to the 1970s. Large population-based cohorts have shown that the incidence 


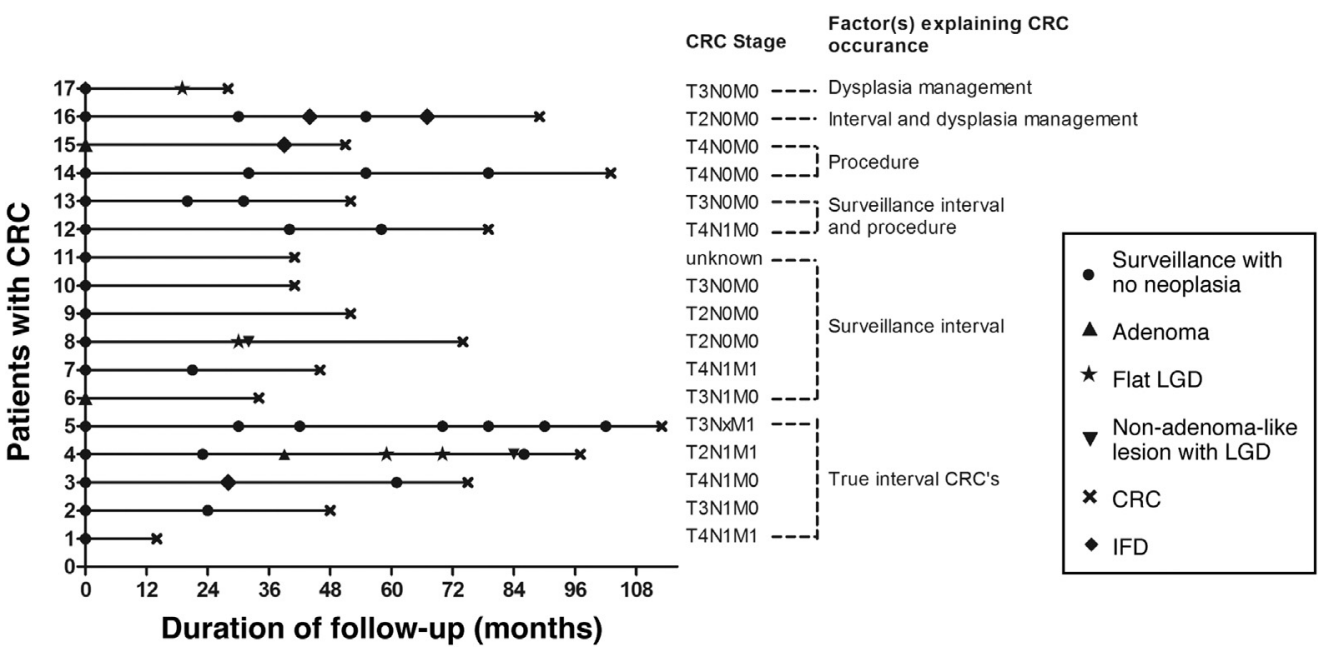

Figure 1. All surveillance colonoscopies and episodes of dysplasia before the diagnosis of CRC. IFD, indefinite for dysplasia. of CRC among IBD patients gradually is decreasing over time, most likely reflecting the improvement in the quality of endoscopes and colonoscopic performance combined with improvements in anti-inflammatory medication, which might well explain the lower incidence of CRC in our study. ${ }^{14,15}$ In addition, the fact that $10 \%$ of surveillance procedures were performed with chromoendoscopy could have improved the dysplasia detection rate and thereby decreased the overall incidence of CRC compared with previous studies.

Only 5 cases of interval CRC, defined as advanced-stage CRC despite adequate surveillance at the appropriate interval before the CRC diagnosis, were identified. Although these CRCs constituted $30 \%$ of the total number of CRCs in

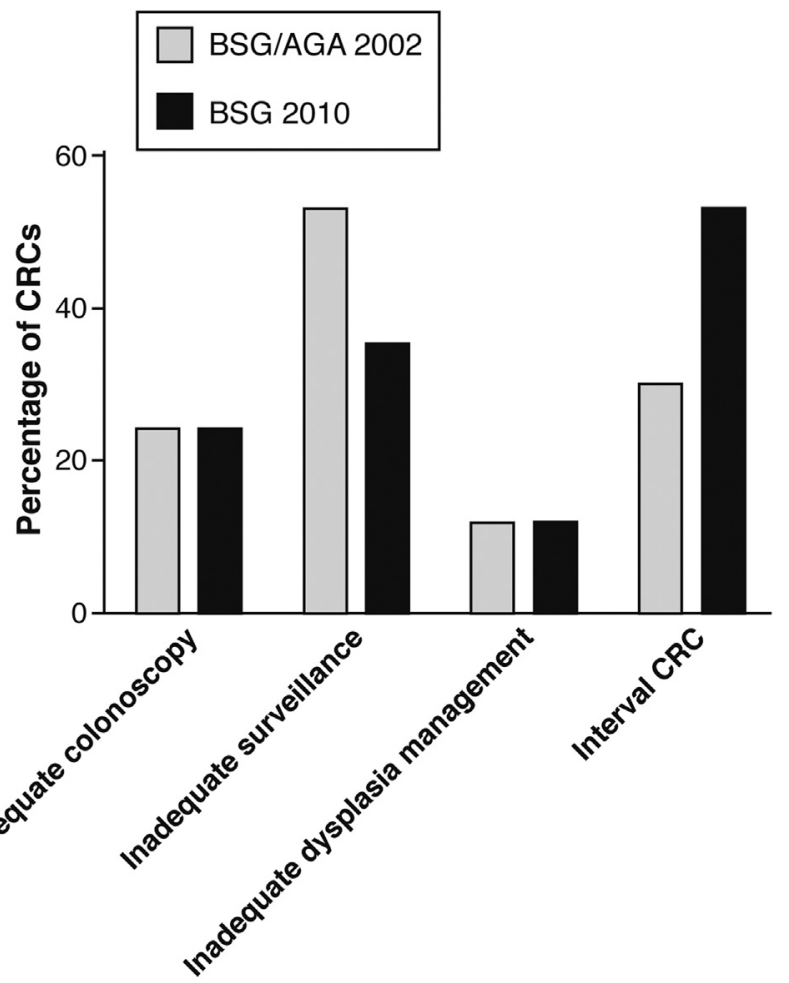

Figure 2. CRC cases subdivided based on the most likely etiologic factor. our cohort, the incidence of 0.7 per 1000 follow-up years was considerably lower than the 2.7 per 1000 follow-up years reported by Rutter et al. ${ }^{8}$ Again, the fact that our data originate from a more recent era with improved endoscopes and most likely a lower overall risk of CRC seems a logical explanation for this discrepancy. Another more recently published study on interval CRCs among IBD patients identified all cases of CRC diagnosed during or within 36 months after colonoscopy and compared the percentage of CRCs diagnosed 6 to 36 months after colonoscopy (interval CRCs) out of the total number of CRCs between patients with and without IBD. ${ }^{16}$ In this study, the percentage of interval CRCs was 3 times higher in IBD patients compared with those without IBD, stressing the importance of close surveillance in these patients. This study lacked data on the colonoscopies before the CRC diagnosis including the number of biopsy specimens, bowel preparation, and the data to assess the appropriate surveillance interval for each patient. Therefore, the number of interval CRCs cannot be compared with our study.

Reports on the incidence of interval CRC (defined as CRC after a negative colonoscopy) among patients without IBD have ranged between 0.2 and 1.2 per 1000 years of follow-up evaluation. ${ }^{17,18}$ The incidence of true interval CRCs in our study of 0.7 per 1000 years of follow-up evaluation therefore is comparable with the interval CRC incidence in patients undergoing surveillance for sporadic CRC.

Two likely explanations for the occurrence of interval CRCs are either missed dysplastic lesions or cancer developing in the time between 2 surveillance colonoscopies. Although it has been suggested that the carcinogenesis in IBD-associated CRC might be accelerated as compared with sporadic CRC, the fact that dysplasia in IBD patients often is flat and therefore can be overlooked more easily offers a plausible explanation for the occurrence of interval CRCs in these patients. ${ }^{19,20}$ The low rate of interval CRC in our cohort suggests that missed lesions do not pose a large problem when surveillance is performed at an interval of 1 to 2 years. Although optimal surveillance intervals cannot be 
deduced from our study, the low incidence of both CRC and interval CRC might justify surveillance intervals of 3 or even 5 years in patients without additional risk factors, as suggested in the updated BSG and European Crohn's and Colitis Organisation guidelines. ${ }^{3,21}$ Unfortunately, we were unable to perform a risk-factor analysis for interval CRCs because of the low number of such cases identified in the current study.

Our study had several limitations that need to be addressed. The low overall incidence of CRC in our surveillance cohort precluded a meaningful analysis of risk factors for interval CRCs.

The retrospective nature of this study resulted in several limitations as well. First, the criteria for inadequate surveillance were based on endoscopy reports rather than a standardized protocol, potentially introducing reporter bias regarding adequate bowel preparation and cecal intubation rate. Another potential confounding factor might have been the classification of lesions as either adenoma-like or non-adenoma-like because this was based on the description of the lesion taken from the endoscopy report.

Furthermore, we were unable to identify the reasons why in some cases the recommended surveillance interval was not used. This information is important because this could guide an intervention toward either better education of patients regarding the need for surveillance or better understanding of the guidelines among gastroenterologists.

In conclusion, this study showed that the incidence of CRC is low among IBD patients undergoing regular endoscopic surveillance. This might support the longer surveillance interval of up to 5 years as recommended in the current BSG and European Crohn's and Colitis Organisation guidelines, although the fact that one third of all CRC cases appear to be interval carcinomas underscores the need to further identify risk factors associated with the development of interval cancer.

\section{References}

1. Ekbom A, Helmick C, Zack M, et al. Ulcerative colitis and colorectal cancer. A population-based study. N Engl J Med 1990; 323:1228-1233.

2. Ekbom A, Helmick C, Zack M, et al. Increased risk of large-bowel cancer in Crohn's disease with colonic involvement. Lancet 1990;336:357-359.

3. Cairns SR, Scholefield JH, Steele RJ, et al. Guidelines for colorectal cancer screening and surveillance in moderate and high risk groups (update from 2002). Gut 2010;59:666-689.

4. Farraye FA, Odze RD, Eaden J, et al. AGA medical position statement on the diagnosis and management of colorectal neoplasia in inflammatory bowel disease. Gastroenterology 2010;138:738-745.

5. Lutgens MW, Oldenburg B, Siersema PD, et al. Colonoscopic surveillance improves survival after colorectal cancer diagnosis in inflammatory bowel disease. $\mathrm{Br} J$ Cancer 2009; 101:1671-1675.

6. Karlen P, Kornfeld D, Brostrom O, et al. Is colonoscopic surveillance reducing colorectal cancer mortality in ulcerative colitis? A population based case control study. Gut 1998; 42:711-714.

7. Sanduleanu S, Rutter MD. Interval colorectal cancers in inflammatory bowel disease: the grim statistics and true stories. Gastrointest Endosc Clin N Am 2014;24:337-348.

8. Rutter MD, Saunders BP, Wilkinson KH, et al. Thirty-year analysis of a colonoscopic surveillance program for neoplasia in ulcerative colitis. Gastroenterology 2006;130:1030-1038.

9. le Clercq CM, Bouwens MW, Rondagh EJ, et al. Postcolonoscopy colorectal cancers are preventable: a population-based study. Gut 2014;63:957-963.

10. Verschuren EC, Ong DE, Kamm MA, et al. Inflammatory bowel disease cancer surveillance in a tertiary referral hospital: attitudes and practice. Intern Med J 2014;44:40-49.

11. Velayos FS, Liu L, Lewis JD, et al. Prevalence of colorectal cancer surveillance for ulcerative colitis in an integrated health care delivery system. Gastroenterology 2010;139:1511-1518.

12. Eaden JA, Ward BA, Mayberry JF. How gastroenterologists screen for colonic cancer in ulcerative colitis: an analysis of performance. Gastrointest Endosc 2000;51:123-128.

13. van Rijn AF, Fockens P, Siersema PD, et al. Adherence to surveillance guidelines for dysplasia and colorectal carcinoma in ulcerative and Crohn's colitis patients in the Netherlands. World J Gastroenterol 2009;15:226-230.

14. Lutgens MW, van Oijen MG, van der Heijden GJ, et al. Declining risk of colorectal cancer in inflammatory bowel disease: an updated meta-analysis of population-based cohort studies. Inflamm Bowel Dis 2013;19:789-799.

15. Beaugerie L, Svrcek M, Seksik P, et al. Risk of colorectal highgrade dysplasia and cancer in a prospective observational cohort of patients with inflammatory bowel disease. Gastroenterology 2013;145:166-175.

16. Wang YR, Cangemi JR, Loftus EV Jr, et al. Rate of early/missed colorectal cancers after colonoscopy in older patients with or without inflammatory bowel disease in the United States. Am J Gastroenterol 2013;108:444-449.

17. Leung K, Pinsky P, Laiyemo AO, et al. Ongoing colorectal cancer risk despite surveillance colonoscopy: the Polyp Prevention Trial Continued Follow-up Study. Gastrointest Endosc 2010;71:111-117.

18. Kaminski MF, Regula J, Kraszewska E, et al. Quality indicators for colonoscopy and the risk of interval cancer. N Engl J Med 2010;362:1795-1803.

19. Ullman TA, Itzkowitz SH. Intestinal inflammation and cancer. Gastroenterology 2011;140:1807-1816.

20. Kiesslich R, Fritsch J, Holtmann M, et al. Methylene blue-aided chromoendoscopy for the detection of intraepithelial neoplasia and colon cancer in ulcerative colitis. Gastroenterology 2003; 124:880-888.

21. Annese V, Daperno M, Rutter MD, et al. European evidence based consensus for endoscopy in inflammatory bowel disease. J Crohns Colitis 2013;7:982-1018.

\section{Reprint requests}

Address requests for reprints to: Bas Oldenburg, MD, PhD, Department of Gastroenterology and Hepatology, University Medical Center Utrecht, Heidelberglaan 100, 3584 CX Utrecht, The Netherlands. e-mail: b.oldenburg@ umcutrecht.nl; fax: (0031) 887555533.

\section{Conflicts of interest}

This author discloses the following: Bas Oldenburg is a consultant for AbbVie $\mathrm{BV}, \mathrm{MSD} \mathrm{BV}$, and Ferring BV. The remaining authors disclose no conflicts. 